\title{
COMPETÊNCIAS FAMILIARES RELACIONADAS À PREVENÇÃO E TRATAMENTO DAS DOENÇAS NAS CRIANÇAS DE ATÉ SEIS ANOS
}

\section{Francisco Ariclene Oliveira}

Graduado em Enfermagem pela Faculdade Metropolitana da Grande Fortaleza (FAMETRO), Bolsista do Programa Bolsa de Incentivo à Educação na Rede SESA (PROENSINO/SESA), Fortaleza(CE), Brasil.

\section{Francisca Jamille Mourão Ximenes}

Graduada em Enfermagem pela Faculdade Metropolitana da Grande Fortaleza (FAMETRO), Fortaleza(CE), Brasil.

\section{Ana Clécia Silva Monteiro}

Graduada em Enfermagem pela Faculdade Maurício de Nassau (UNINASSAU), Fortaleza(CE), Brasil.

\section{Odaleia de Oliveira Farias}

Mestranda do Programa de Pós-graduação em Enfermagem da Universidade de Federal do Ceará (UFC), Fortaleza(CE), Brasil.

\section{Igor Cordeiro Mendes}

Doutorando do Programa de Pós-graduação em Enfermagem da Universidade de Federal do Ceará (UFC), Fortaleza(CE), Brasil.

\section{Cristiana Ferreira da Silva}

Doutora em Saúde Coletiva pela Universidade Federal do Ceará (UFC), Docente da Faculdade Metropolitana da Grande Fortaleza (FAMETRO), Fortaleza(CE), Brasil.

\section{Denizielle de Jesus Moreira Moura}

Doutora em Cuidados Clínicos em Saúde pela Universidade Estadual do Ceará (UECE), Docente da Faculdade Metropolitana da Grande Fortaleza (FAMETRO), Fortaleza(CE), Brasil.
RESUMO: Objetivou-se avaliar as competências familiares relacionadas à prevenção $\mathrm{e}$ ao tratamento das doenças nas crianças de até seis anos de idade cadastradas em uma Unidade de Atenção Primária à Saúde da Estratégia Saúde da Família de Fortaleza (CE). Trata-se de um estudo transversal, descritivo e quantitativo. Os dados foram coletados nos meses de fevereiro a maio de 2017, envolvendo uma amostra de 217 famílias. Os resultados demonstraram que $98,2 \%$ das crianças estão com o calendário vacinal básico atualizado. Identificou-se que $67,7 \%$ dos cuidadores lavam as mãos das crianças depois de ir ao banheiro e $76,0 \%$ adotam essa medida de higiene antes de comer algum alimento. Verificou-se que mais de $90 \%$ das famílias identificam sinais comuns de adoecimento que requerem tratamento em hospitais e levam as crianças para esses serviços. Nessa perspectiva, acredita-se que a Estratégia Saúde da Família é um importante espaço de produção de saúde e de fortalecimento das competências familiares da população adscrita.

PALAVRAS-CHAVE: Família; Prevenção de doenças; Estratégia saúde da família.

\section{FAMILY COMPETENCE RELATED TO PREVENTION AND TREATMENT OF DISEASES IN UP TO SIX-YEAR-OLD CHILDREN}

\begin{abstract}
Current study evaluates family competence related to the prevention and treatment of disease in up to six-year-old children enrolled in a Basic Health Care Unit for Family Health Strategy in Fortaleza CE Brazil. The transversal, descriptive and quantitative study comprised data retrieved between February and May 2017, from a sample of 217 families. Results showed that $98.2 \%$ of the children received basic vaccine and that $67.7 \%$ of caretakers wash children's hands after toilet use and $76.0 \%$ wash their hands prior to feeding. More than $90 \%$ of families identified common signs for diseases that needed treatment in hospitals. Within this perspective, Family Health Strategy is an important element for health production and improvement within family competences of the population.
\end{abstract}

KEY WORDS: Family; Prevention of diseases; Family health strategy.

\section{INTRODUÇÃO}

O desenvolvimento começa desde a concepção, sendo a primeira infância um período de particular vulnerabilidade a fatores de risco. As experiências mais formativas nos primeiros anos vêm dos pais,
Autor correspondente

Francisco Ariclene Oliveira

E-mail: franciscoariclene@hotmail.com 
dos familiares, de cuidadores e da comunidade. Estudos recentes sobre desenvolvimento infantil corroboram que esse processo resulta da interação do ser com seu mundo: sua classe social, seu local de vida e época ${ }^{1}$. Assim, entende-se que uma criança com bom desenvolvimento está inserida em um ambiente estável, que é sensível à saúde das crianças e suas necessidades nutricionais, com proteção contra ameaças, com oportunidades de aprendizagem precoce, garantindo apoio emocional e um desenvolvimento estimulante.

Famílias que garantem à criança uma boa alimentação, acesso à saúde, estimulação precoce adequada e que oportunizam um ambiente familiar acolhedor, favorecem que estas crianças venham a ser adultos mais produtivos do que aqueles que não tiveram esses fatores $^{2}$. Daí a importância de investir nos primeiros anos de vida do ser humano, haja vista as potenciais contribuições para o desenvolvimento saudável da criança.

A família, como primeira base formadora, na qual a criança tem contato, tem oportunidade privilegiada para contribuir positivamente no processo de desenvolvimento de suas crianças, garantindo, assim, os direitos fundamentais destas. No entanto, para que a família cumpra bem o seu papel, ela precisa ser apoiada e fortalecida. Essa tarefa exige o aprimoramento de comportamentos, habilidades e atitudes, as quais a Estratégia Saúde Família (ESF) pode atuar para fortalecê-las em seu território, afirmando ser um importante espaço de fortalecimento das competências parentais, haja vista o acompanhamento que os profissionais de saúde fazem das famílias, além do conhecimento da realidade da população da área adscrita $^{3}$.

A Estratégia da Saúde Família, criada pelo Ministério da Saúde, em 1994, com atuação marcante da equipe multidisciplinar, surge com o intuito de priorizar as ações de prevenção, promoção e recuperação da saúde. Nessa concepção, o Agente Comunitário de Saúde (ACS) se apresenta como uma referência para a população em sua área de atuação, apresentando posição estratégica de alcance às famílias, sendo um importante facilitador na identificação dos agravos da comunidade, na orientação e monitoramento das famílias e na realização de atividades domiciliares e no fortalecimento de ações de promoção à saúdé .
Desse modo, é imprescindível que sejam constantemente avaliadas as competências familiares na atenção à saúde das crianças de até seis anos de vida, com vistas a conhecer falhas de competências essenciais ao desenvolvimento infantil saudável, possibilitando ações que visem promover comportamentos que assegurem seu pleno desenvolvimento físico e mental. As competências familiares são compreendidas como o conjunto dos conhecimentos, saberes e habilidades que aliados à afetividade, às atitudes e às práticas das famílias favorecem a sobrevivência, o desenvolvimento, a proteção e a participação das crianças nas decisões importantes para ela ${ }^{3}$.

Diante desse contexto, considera-se que a avaliação das competências familiares na atenção à saúde das crianças de até seis anos é fundamental para a tomada de decisões. Nessa perspectiva, objetivou-se nesse estudo avaliar as competências familiares relacionadas à prevenção e ao tratamento das doenças nas crianças de até seis anos de idade cadastradas em uma Unidade de Atenção Primária à Saúde (UAPS) da Estratégia Saúde da Família de Fortaleza.

\section{METODOLOGIA}

Trata-se de um estudo transversal, de natureza descritiva e abordagem quantitativa. Os dados foram coletados no período de fevereiro a maio de 2017, na área adscrita de uma Unidade de Atenção Primária à Saúde, no âmbito da Estratégia Saúde da Família, compreendida na Coordenadoria Regional de Saúde - CORES V, do Município de Fortaleza (CE).

Nesse estudo, optou-se pela amostragem por conveniência, na qual os ACS das microáreas sorteadas foram selecionando os participantes até atingir a amostra de 217 famílias. Essa amostra foi determinada a partir do cálculo amostral para população finita com uma prevalência média esperada de até 50\% para diversos indicadores, com nível de confiança de $95 \%$ e com margem de erro de $5 \%$.

Os critérios de inclusão das famílias avaliadas nesse estudo foram residir na área de acompanhamento da ESF e possuir pelo menos uma criança de até seis anos de idade. Nos casos em que a família tinha mais de uma criança, levou-se em conta os dados da criança mais 
velha, considerando-se que os cuidados oferecidos às demais crianças seriam semelhantes.

Utilizou-se para a coleta de dados um formulário padronizado e pré-codificado, com perguntas fechadas, estruturado com dez questões/indicadores, contemplando quatro competências familiares, elaborado e testado pelo Fundo das Nações Unidas para Infância (Unicef), cuja finalidade foi investigar aspectos relativos ao tratamento e à prevenção de doenças comuns na primeira infância, a posse da caderneta de vacinação e se o responsável pelos cuidados da criança sabe interpretar corretamente o gráfico de peso/idade; além de investigar o conhecimento sobre sinais indicativos de gravidade de doença na infância e a adoção de medidas de higiene e de cuidados para prevenir malária ou dengue.

A aplicação do formulário foi desenvolvida pelos pesquisadores e por três ACS. Estes profissionais foram escolhidos pelo fato de conhecerem a comunidade, facilitando o acesso às famílias, reduzindo a recusa da participação na pesquisa. Além disso, acredita-se que a participação dos agentes no treinamento da pesquisa pode servir como uma primeira oportunidade para sensibilizá-los sobre a importância do fortalecimento das competências familiares voltadas à atenção da criança na primeira infância. Ressalta-se que a coleta dos dados foi realizada no domicílio do participante, mantendo a privacidade e a liberdade de resposta do responsável/cuidador.

Os entrevistadores selecionados receberam treinamento de dois dias sobre técnicas de entrevistas espe- cíficas para as competências familiares. O treinamento constou da apresentação da pesquisa, leitura do questionário e simulação.

Os dados obtidos foram digitados e processados utilizando-se as planilhas eletrônicas do programa Microsoft Office Excel@ 2016. Após tabulados, os dados foram calculados e estudados, extraindo as frequências absolutas e percentuais. Diante da consolidação dos resultados, a análise dos dados foi realizada à luz da literatura científica e comparando a outros estudos.

O estudo respeitou todos os princípios éticos da resolução n ${ }^{\circ}$. 510/16 do Conselho Nacional de Saúde que regulamenta a pesquisa com seres humanos no país ${ }^{5}$. A pesquisa encontra-se aprovada pelo Comitê de Ética e Pesquisa da Secretaria de Saúde do Estado do Ceará - SESA/CE, sob parecer de $n^{\circ}$. 1.427.214 e CAAE $n^{\circ}$. 52247115.6.0000.5051.

\section{RESULTADOS}

Os dados apresentados, nas tabelas a seguir, estão dispostos na sequência do formulário aplicado aos entrevistados. As tabelas estão organizadas por indicadores que são bases para aferição das competências familiares. A Tabela 1 sintetiza os dados a respeito das competências familiares e dos indicadores/questões referentes à prevenção e ao tratamento das doenças das crianças.

Tabela 1. Competência familiar e indicadores de saúde relacionados à prevenção das doenças das crianças de até seis anos. Fortaleza, 2017

(Continua)

\begin{tabular}{|c|c|c|c|c|}
\hline COMPETÊNCIA FAMILIAR & INDICADORES & RESPOSTAS & $\mathbf{N}$ & $\%$ \\
\hline \multirow{2}{*}{ - } & \multirow{2}{*}{ A criança tem a caderneta de vacinação. } & Sim & 213 & 98,2 \\
\hline & & Não & 4 & 1,8 \\
\hline \multirow{3}{*}{-} & \multirow{3}{*}{$\begin{array}{l}\text { A mãe mostrou a caderneta de vaci- } \\
\text { nação. }\end{array}$} & Sim, mostrou. & 177 & 81,6 \\
\hline & & Não mostrou. & 36 & 16,6 \\
\hline & & NSA & 4 & 1,8 \\
\hline \multirow{3}{*}{-} & \multirow{3}{*}{$\begin{array}{l}\text { Os profissionais estão registrando as } \\
\text { informações de saúde da criança. }\end{array}$} & Vacinas & 206 & 95,0 \\
\hline & & Peso & 195 & 89,9 \\
\hline & & Desenvolvimento & 183 & 84,3 \\
\hline
\end{tabular}


Conclusão)

\begin{tabular}{|c|c|c|c|c|}
\hline COMPETÊNCIA FAMILIAR & INDICADORES & RESPOSTAS & $\mathbf{N}$ & $\%$ \\
\hline \multirow{4}{*}{$\begin{array}{l}\text { A família providencia para que } \\
\text { a criança esteja completamente } \\
\text { imunizada, de acordo com sua } \\
\text { faixa etária. }\end{array}$} & \multirow{2}{*}{$\begin{array}{l}\text { Crianças completamente imunizadas } \\
\text { para a sua idade. }\end{array}$} & $\operatorname{Sim}$ & 209 & 96,3 \\
\hline & & Não & 8 & 3,7 \\
\hline & \multirow{2}{*}{$\begin{array}{l}\text { A mãe/responsável recebeu orientação } \\
\text { sobre o peso e o desenvolvimento pe- } \\
\text { los profissionais da ESF. }\end{array}$} & Sim & 192 & 88,5 \\
\hline & & Não & 25 & 11,5 \\
\hline \multirow{4}{*}{ - } & \multirow{4}{*}{$\begin{array}{l}\text { A mãe/responsável reconhece o perfil } \\
\text { de crescimento no gráfico do cartão de } \\
\text { vacinas. }\end{array}$} & Está crescendo bem. & 197 & 90,8 \\
\hline & & Tem dúvida. & 15 & 6,9 \\
\hline & & Não está crescendo bem. & 1 & 0,5 \\
\hline & & Não sabe. & 4 & 1,8 \\
\hline
\end{tabular}

Em relação à caderneta de Saúde da Criança, observou-se que $98,2 \%$ das crianças consideradas no estudo tinham esse documento tão importante para o registro e o acompanhamento de saúde. Deste percentual, 81,6\% dos responsáveis mostraram a caderneta para os entrevistadores ao serem solicitadas para averiguação. Ao verificar os registros feitos pelos profissionais de saúde sobre as vacinas, acompanhamento do peso e do desenvolvimento da criança, observou-se que $95,0 \%$ dos cartões apresentavam registros adequados das vacinas para a idade da criança, ao passo que as anotações do peso e o do registro do desenvolvimento infantil foram registrados em $89,9 \%$ e $84,3 \%$ das cadernetas, respectivamente.

Analisando os dados da criança acerca do registro de vacinas, constatou-se que $96,3 \%$ das crianças estão com o calendário vacinal básico atualizado de acordo com sua idade, diminuindo a vulnerabilidade dos mesmos às doenças imunopreveníveis.
Ao questionar se os profissionais da ESF - médico, enfermeiro e/ou ACS - explicam e orientam aos responsáveis acerca de como está o peso e o desenvolvimento da criança, identificou-se que $88,5 \%$ dos cuidadores recebem essas orientações. No entanto, observa-se um percentual significativo de profissionais que apenas registram os indicadores sem comentar o que significam aos pais.

Ao solicitar ao cuidador que observasse o gráfico de crescimento na caderneta da criança com intuito de verificar se ele reconhecia o perfil de crescimento, identificou-se que a grande maioria compreendia a evolução do registro do gráfico de crescimento, sendo que $90,8 \%$ afirmaram que a criança estava com bom crescimento e apenas $8,7 \%$ tinham dúvidas ou não sabiam explicar. 
Tabela 2. Competência familiar relacionada ao tratamento da criança doente. Fortaleza, 2017

\begin{tabular}{|c|c|c|c|c|}
\hline $\begin{array}{l}\text { COMPETÊNCIA } \\
\text { FAMILIAR }\end{array}$ & INDICADORES & RESPOSTAS & $\mathbf{N}$ & $\%$ \\
\hline \multirow{14}{*}{$\begin{array}{l}\text { A família reconhece quan- } \\
\text { do a criança doente precisa } \\
\text { de tratamento em hospitais } \\
\text { ou centros de saúde e a leva } \\
\text { para esses serviços. }\end{array}$} & \multirow{14}{*}{$\begin{array}{l}\text { Famílias que recon- } \\
\text { hecem pelo menos dois } \\
\text { sinais que requerem } \\
\text { tratamento em hospitais } \\
\text { ou centro de saúde e } \\
\text { leva a criança para esses } \\
\text { serviços. }\end{array}$} & Criança tem ataque, convulsão & 217 & 100,0 \\
\hline & & Criança tem tosse com febre alta & 216 & 99,5 \\
\hline & & Criança tem sangue nas fezes & 215 & 9,1 \\
\hline & & Criança tem vômito & 214 & 98,6 \\
\hline & & Criança fica cada vez mais doente & 214 & 98,6 \\
\hline & & Criança fica com os lábios, pés e mãos arroxeados & 212 & 97,7 \\
\hline & & Criança tem diarreia & 210 & 96,8 \\
\hline & & Criança fica com febre & 209 & 96,3 \\
\hline & & Criança respira com dificuldade & 208 & 95,8 \\
\hline & & $\begin{array}{l}\text { Criança tem olhos fundos, sede intensa, moleza } \\
\text { extrema }\end{array}$ & 208 & 95,8 \\
\hline & & Criança fica com a pele muito amarelada & 203 & 93,5 \\
\hline & & Criança respira rápido com chiado & 193 & 89,0 \\
\hline & & Criança bebe pouco líquido & 141 & 65,0 \\
\hline & & Criança não consegue nem mamar no peito & 133 & 61,3 \\
\hline
\end{tabular}

Com relação às famílias que reconhecem sinais que requerem tratamento em hospitais ou centro de saúdes e que levam as crianças para esses serviços, mais de 90\% dos respondentes identificaram positivamente sinais comuns de adoecimento na criança, tais como pele ama- relada, febre, dispneia, melena, diarreia, vômito, tosse e convulsão. No entanto, sinais considerados também graves, como chiado no peito e desidratação foram reconhecidos por $89,9 \%$ e $65,0 \%$, respectivamente.

Tabela 3. Competências familiares e indicadores de saúde relacionados à prevenção de doença em crianças de até seis anos. Fortaleza, 2017

(Continua)

\begin{tabular}{|c|c|c|c|c|}
\hline $\begin{array}{l}\text { COMPETÊNCIA } \\
\text { FAMILIAR }\end{array}$ & INDICADORES & RESPOSTAS & $\mathbf{N}$ & $\%$ \\
\hline \multirow{4}{*}{$\begin{array}{l}\text { Os membros da família lavam } \\
\text { as mãos antes da preparação } \\
\text { de alimentos, de alimentar a } \\
\text { criança e principalmente após } \\
\text { a defecação. }\end{array}$} & \multirow{4}{*}{$\begin{array}{l}\text { Membros da família que } \\
\text { lavam as mãos antes do } \\
\text { preparo da comida e } \\
\text { antes de alimentar a cri- } \\
\text { ança }\end{array}$} & Antes de cozinhar & 204 & 94,4 \\
\hline & & Depois de ir ao banheiro & 191 & 88,0 \\
\hline & & $\begin{array}{l}\text { Depois de trocar as fraldas ou levar a criança ao } \\
\text { banheiro }\end{array}$ & 141 & 65,0 \\
\hline & & Antes de dar comida para a criança & 113 & 88,0 \\
\hline \multirow{2}{*}{ - } & \multirow{2}{*}{$\begin{array}{l}\text { Ocasiões em que a cri- } \\
\text { ança costuma lavar as } \\
\text { mãos ou são lavadas. }\end{array}$} & Depois de ir ao banheiro & 147 & 67,7 \\
\hline & & Antes de comer & 165 & 76,0 \\
\hline
\end{tabular}




\begin{tabular}{|c|c|c|c|c|}
\hline $\begin{array}{l}\text { COMPETÊNCIA } \\
\text { FAMILIAR }\end{array}$ & INDICADORES & RESPOSTAS & $\mathbf{N}$ & $\%$ \\
\hline \multirow{8}{*}{$\begin{array}{l}\text { A família protege a criança e } \\
\text { gestantes em áreas atingidas } \\
\text { por malária e/ou por dengue. }\end{array}$} & \multirow{8}{*}{$\begin{array}{l}\text { Cuidados para prevenir } \\
\text { dengue e malária }\end{array}$} & $\begin{array}{l}\text { Mantêm os recipientes com água sempre tampa- } \\
\text { dos }\end{array}$ & 204 & 94,0 \\
\hline & & Não deixa pneus no quintal & 156 & 71,9 \\
\hline & & Quintal sem garrafas vazias ou sacos plásticos & 195 & 89,9 \\
\hline & & Manutenção do quintal limpo & 73 & 79,7 \\
\hline & & Uso de mosquiteiro & 114 & 52,6 \\
\hline & & Uso de repelente fora de casa & 95 & 43,8 \\
\hline & & Uso de telas nas janelas e portas & 31 & 14,3 \\
\hline & & Não tomar banho de rio ao anoitecer & 17 & 7,9 \\
\hline
\end{tabular}

As práticas de higiene adotadas pelos cuidadores repercutem diretamente na saúde das crianças, a respeito da prática da lavagem das mãos, sondaram-se em quais ocasióes os responsáveis haviam lavado as mãos no dia anterior; obtendo-se as seguintes respostas: antes de cozinhar $(94,4 \%)$; de dar comida para a criança $(88,0 \%)$; o percentual que o fizeram depois de trocar a fralda da criança e de ir ao próprio banheiro foi bem menor, sendo $65,0 \% ; 88,0 \%$, nesta ordem.

No que se refere à higienização das mãos das crianças, identificou-se um dado preocupante, haja vista que apenas $67,7 \%$ lavam as mãos depois de ir ao banheiro e $76,0 \%$ adotam essa medida de higiene ante de comer algum alimento.

Embora todos os domicílios pesquisados possuam água encanada e banheiro, o nível de higienização dos cuidadores e das crianças ainda não é uma prática universal, evidenciando que há descuido de higiene, desde o uso do sanitário até no preparo da comida da criança, prática que leva ao desenvolvimento de doenças que poderiam ser evitadas, como verminoses, diarreias e infecções intestinais, entre outras.

Considerando os cuidados da família para prevenir malária e dengue, observou-se que as medidas mais adotadas foram deixar os recipientes com água tampados (94,0\%), seguido da manutenção de quintais/terrenos limpos sem garrafa ou sacos plásticos (89,9\%), mesmo sendo uma atitude simples e acessível, a utilização de mosquiteiro só foi reportada por cerca de 53,0\% dos responsáveis e sendo disponível apenas às crianças.

\section{DISCUSSÃO}

A pesquisa mostrou que $98,2 \%$ das crianças tinham a caderneta de vacinação, evidenciando que os serviços de saúde estão garantindo esse direito às famílias e estas reconhecem a importância desse documento tão importante para o registro e o acompanhamento de saúde da criança. Os demais responsáveis que disseram não ter o documento justificaram a perda do mesmo ou que por falta de cuidados o mesmo havia se deteriorado, comprometendo as anotações, de modo que essas crianças contam apenas com os registros do cartão-espelho dos ACS da área. A Caderneta de Saúde da Criança (CSC) é um instrumento essencial de vigilância à saúde e seu uso correto favorece a comunicação, a educação em saúde e a promoção da saúde infantil ${ }^{6}$.

A CSC destina-se a todas as crianças nascidas no Brasil e reúne o registro dos mais importantes eventos relacionados à saúde da criança. Ressalta-se que o preenchimento deve ser iniciado ainda na maternidade com o registro das informações acerca do parto, condições de alta do bebê, primeiras vacinas e exames ou testes realizados, e sua continuidade deve ser dada, preferencialmente, pelos profissionais da Estratégia Saúde da Família ${ }^{7}$.

Certificou-se que 96,3\% das crianças estão com o calendário vacinal básico atualizado de acordo com sua idade, este dado se apresenta como um fator positivo de proteção contra as doenças imunopreveníveis. Desse 
modo, considera-se que a situação vacinal das crianças da pesquisa é satisfatória para todas as vacinas do Programa Nacional de Imunização - PNI, tendo em vista que tal resultado se encontra acima do estabelecido pelo Ministério da Saúde, cuja recomendação é que devem estar em torno de $90 \%$ a $100 \%$ para um controle efetivo das doenças imunopreveníveis ${ }^{8}$.

Nessa perspectiva, acredita-se que a compreensão pelos pais/responsáveis da importância da vacinação infantil é fundamental para a adesão ao esquema vacinal completo. Por isso, faz-se necessário o desenvolvimento de ações de orientação aos pais sobre a relevância de imunizar a criança, de forma a conscientizá-los, e também estimular a formação de programas de intervenção para diminuir cada vez mais o número de crianças não imunizadas?.

Verificou-se que $95,0 \%$ dos cartões apresentam o registro adequado das vacinas, no entanto observou que as anotações do peso foram registradas em $89,9 \%$ das cadernetas, resultado semelhante a um estudo com crianças de Belo Horizonte/MG, no qual constatou que o peso estava registrado corretamente em $91 \%$ das cadernetas de vacina ${ }^{10}$. Para que a caderneta da criança cumpra seu papel de instrumento de comunicação, vigilância e promoção da saúde infantil é fundamental sua utilização adequada, o que inclui o registro correto e completo das informações, além do diálogo com a família sobre as anotações nele realizadas.

A grande maioria dos entrevistados relatou que recebe orientações referentes ao peso e ao desenvolvido da criança dos profissionais da ESF. A respeito desse resultado, verificou-se que $11,5 \%$ profissionais registram os indicadores de crescimento e desenvolvimento sem explicá-los aos responsáveis a implicação desses indicadores para a saúde da criança. Considerando esses resultados, faz-se necessário que as equipes da saúde da família realizem o acompanhamento do desenvolvimento das crianças nos dois primeiros anos de vida, haja vista que este monitoramento é compreendido como o eixo integrador das ações básicas por meio da sistematização do retorno do RN e da criança à unidade de saúde, sendo desse modo um indicador sensível e positivo de saúde, dentro de uma concepção integral ${ }^{11}$.

No que se refere à compreensão do gráfico de crescimento na caderneta da criança, identificou-se no estudo que $90,8 \%$ dos participantes, ao analisarem o gráfi- co, afirmaram que a criança estava com bom crescimento e apenas $8,7 \%$ tinham dúvidas ou não sabiam explicar o perfil de crescimento. Diante desses resultados, acreditase que as orientações recebidas pelos profissionais podem refletir diretamente nesse expressivo percentual de modo positivo. Nesse sentido, reforça-se a importância do acompanhamento do crescimento, haja vista que esse indicador permite avaliar o desenvolvimento da criança, identificando aquelas com maior risco de morbimortalidade, prevenindo precocemente a desnutrição e promovendo o crescimento, favorecendo, assim, a melhor qualidade de vida para a população infantil ${ }^{12}$.

Com relação às famílias que reconhecem sinais de alerta para a busca de atenção médica em hospitais ou centro de saúdes e que levam as crianças para esses serviços, mais de $90 \%$ dos respondentes identificaram positivamente os sinais mais comuns de adoecimento da criança, sendo eles: pele amarelada, febre, dispneia, melena (sangue nas fezes), diarreia, vômito, tosse e convulsão. Resultados semelhantes a de um estudo desenvolvido com famílias de um município baiano, no qual apresentou variação nesses indicadores entre $92,0 \%$ a 93,8\% ${ }^{13}$.

No entanto, sinais considerados também graves, como chiado no peito e desidratação foram reconhecidos por $89,9 \%$; $65,0 \%$, respectivamente. Os resultados apresentados denotam que essas famílias precisam de mais esclarecimentos para saber reconhecer os sinais de adoecimento que demandam urgência hospitalar, para isso seria importante a parceria da ESF com as escolas, creches e pré-escolas da comunidade para intervenção dessa realidade.

Ressalta-se que esses dados evidenciam a vulnerabilidade que essas crianças têm, pelo risco de uma busca tardia pelos serviços de saúde e a falta de uma intervenção precoce. Em face dessa realidade, vem à tona novamente a atuação dos profissionais da estratégia saúde da família, em particular dos ACS, cuja finalidade é promover o acompanhamento sistemático das crianças menores de cinco anos, a fim de desenvolver o vínculo com as famílias, acolhimento, corresponsabilidade e alto grau de resolutividade dos serviços ${ }^{14}$.

Sobre as práticas de higiene do cuidador, identificou-se um percentual expressivo de entrevistados que lava as mãos antes de cozinhar e de dar comida para a 
criança e após sair do banheiro, porém 65,0\% assumiram não o fazer depois de trocar a fralda da criança. Estudos evidenciam que o nível de escolaridade dos responsáveis implica diretamente nesse hábito, assim faz-se necessário que orientações sobre a importância da higienização tanto da mãe, quanto da criança sejam considerados no momento do pré-natal ${ }^{15}$.

A higienização das mãos mostra-se efetiva na prevenção de doenças em crianças e um dos principais elementos de prevenção de contaminação de parasitas intestinais, como os protozoários, platelmintos e nematódeos que acometem, principalmente, crianças pela via oral-fecal ${ }^{16}$.

Ressalta-se que as principais medidas adotadas pelas famílias pesquisadas para prevenir dengue foram: tampar os recipientes com água, limpar quintais/terrenos, mantendo-os sem garrafa ou sacos plásticos. A utilização de mosquiteiro só é utilizada em cerca de 53,0\% dos domicílios e disponíveis apenas às crianças na sua grande maioria. Nesse sentido, é preciso estimular a participação popular junto às instituições, no planejamento das atividades que objetivam a prevenção e o controle da dengue por meio da educação em saúde, fortalecendo o vínculo e criando uma abordagem que vise trabalhar a necessidade da comunidade ${ }^{17}$.

\section{CONCLUSÃO}

Em relação ao desafio de avaliar as competências familiares relacionadas à prevenção e ao tratamento das doenças em crianças de até seis anos de idade, considera-se que o conhecimento dessas competências seja de grande valia para compreender a realidade da população adscrita em relação à atenção que as crianças na primeira infância estão recebendo de seus cuidadores/responsáveis. Ressalta-se, portanto, que a identificação de déficits no que se refere às competências familiares relacionados à prevenção e tratamento das doenças possibilita a elaboração e implementação de ações e estratégias que fortaleçam tais competências.

Os resultados desse estudo demonstraram que as crianças estão com o calendário vacinal básico atualizado de acordo com sua idade, diminuindo a vulnerabilidade das mesmas às doenças imunopreveníveis. No entanto, ao questionar se os profissionais da ESF - médico, enfermeiro e/ou ACS - explicam e orientam aos responsáveis como está o peso e do desenvolvimento da criança, identificou-se que um percentual significativo de entrevistados que relataram não receber tais informações, pois os profissionais apenas registram os indicadores sem comentar aos pais.

O estudo evidenciou ainda a deficiência das famílias na condução da melhor maneira de atender as condições de higiene e da assistência às doenças prevalentes na infância. Ficando clara a necessidade de ampliar e elaborar estratégias visando o fortalecimento das famílias quanto aos cuidados oferecidos às suas crianças.

Ao concluir o estudo, identificou-se que o formulário disponibilizado pelo UNICEF apresenta alguns indicadores que não são condizentes para aplicação às famílias de grandes centros urbanos. Contudo, o presente estudo deve ser realizado em outros lócus de realidade socioeconômicas distintos para comparar as diversas realidades no que diz respeito à compreensão das competências familiares.

Neste sentido, a Estratégia Saúde da Família se apresenta como importante espaço de produção de saúde e de elemento potente para fortalecimento das competências familiares da sua população adscrita. Acreditase, portanto, que estudar e promover o fortalecimento das competências familiares em áreas mais vulneráveis socioeconomicamente, ou até mesmo naquelas onde as condições sociais podem ser mais favoráveis, é urgente e de extrema importância para o desenvolvimento infantil saudável.

\section{REFERÊNCIAS}

1. Eyken EDV, Ribeiro CDM. Desenvolvimento infantil: seus agentes e as políticas públicas do município do Rio de Janeiro. Physis. 2012;22(3):1085-99.

2. Carvalho RS. O investimento na formação do cidadão do futuro: a aliança entre economia e educação infantil como estratégia da governamentalidade contemporânea. Educ rev, 2016;32(2):229-53. 
3. Fundo das Nações Unidas para a Infância. Como avaliar as competências familiares na atenção às crianças de até 6 anos. Brasília (DF): UNICEF; 2005.

4. Moreira TR, Zandonade E, Maciel ELN. Risk of tuberculosis infection among community health agents. Rev Saúde Pública. 2010;44(2):332-8.

5. Ministério da Saúde (BR). Conselho Nacional de Saúde. Resolução n. ${ }^{\circ}$ 510, de 07 de abril de 2016. Aprova normas regulamentadoras que trata das especificidades éticas das pesquisas nas ciências humanas e sociais e de outras que se utilizam de metodologias próprias dessas áreas. Brasília DF: Diário Oficial da União; 2016.

6. Salles IC, Toriyama ATM. A Utilização da Caderneta de Saúde da Criança por Alunos de Enfermagem. Rev Grad USP. 2017;2(2):41-6.

7. Palombo CNT, Duarte LS, Fujimori E, Toriyama ÁTM. Uso e preenchimento da caderneta de saúde da criança com foco no crescimento e desenvolvimento. Rev Esc Enferm USP. 2014;48(esp.):60-72.

8. Lemos EO, Pedrosa DR, Raniéri PSG, Pires CAA, Queiroz AM. Avaliação do cumprimento do calendário de vacinação dos adolescentes de uma escola municipal. Adolesc Saude. 2013:10(2):23-9.

9. Sousa CJ, Vigo ZL, PAlmeira CS. Compreensão dos pais acerca da importância da vacinação infantil. Rev Enferm Contemp, 2012;1(1):44-58.

10. Goulart LMHF, Alves CRL, Viana MRA, Moulin ZS, Carmo GAA, Costa JGDC, et al. Caderneta de Saúde da Criança: avaliação do preenchimento dos dados sobre gravidez, parto e recém-nascido. Rev Paul Pediatr. 2008;26(2):106-12.

11. Ministério da Saúde (BR). Portaria n. 1920, de 5 de setembro de 2013. Institui a Estratégia Nacional para Promoção do Aleitamento Materno e Alimentação Complementar Saudável no Sistema Único de Saúde (SUS) - Estratégia Amamenta e Alimenta Brasil. Brasília (DF): Diário Oficial da União; 2013.

12. Reichert APS, Almeida AB, Souza LC, Silva MEA, Collet
N. Vigilância do crescimento infantil: conhecimento e práticas de enfermeiros da atenção primária à saúde. Rev Rene. 2012;13(1):114-26.

13. Fundo das Nações Unidas para a Infância. Modos de cuidar: dever de casa, do Estado e da sociedade. Salvador (BA): UNICEF; 2008.

14. Souza RS, Ferrari RAP, Santos TFM, Tacla MTGM. Atenção à saúde da criança: prática de enfermeiros da saúde da família. Rev Min Enferm. 2013;17(2):331-9.

15. Castilho ARF. Mialhe FL, Barbosa TS, Puppin-Rontani RM. Influência do ambiente familiar sobre a saúde bucal de crianças: uma revisão sistemática. J Pediatr. 2013;89(2):116-23.

16. Lopes RM, Melo TL. Percepção dos alunos, em anos iniciais do ensino fundamental, relacionada à higienização das mãos. Rev Eletr Interd. 2014;11(1):117-21.

17. Silva IB, Mallmann DG, Vasconcelos EMR. Estratégias de combate à dengue através da educação em saúde: uma revisão integrativa. Saúde. 2015;41(2):27-34.

Recebido em: 23/09/2017 Aceito em: 17/04/2018 